\title{
DINÁMICA FLUVIAL DE UN RÍO DE MONTAÑA PIRENAICO, EL ARA; SU INTERRELACIÓN CON LAS COMUNIDADES VEGETALES
}

\author{
POR \\ V. RUBIO FERNÁNDEZ
}

Y

\section{ANTONIO FERNÁNDEZ FERNÁNDEZ}

\section{Introducción}

En este trabajo se estudia la cuenca del río Ara, situado al Norte de la provincia de Huesca, y que constituye el área de contacto entre los valles occidentales y orientales del Pirineo Aragonés (figura 1). Este río pirenaico nace en el Macizo del Vignemale (Pirineo Axil), factor geográfico que condiciona su régimen nivo-pluvial. Con $688 \mathrm{~km}^{2}$ de cuenca, las aguas discurren desde el valle de Bujaruelo hasta llegar a la Depresión Media. El área de estudio comprende desde la localidad de Broto hasta la confluencia con el río Cinca en Ainsa.

El valle del río Ara registra una notable actividad geomorfológica como consecuencia de la deleznabilidad de los materiales del flysch eoceno que conforman la cuenca, el gran desnivel de las vertientes y la intervención antrópica. Las laderas producen gran cantidad de sedimentos

V. Rubio Fernández. Universidad Autónoma de Madrid (U.A.M.).

Antonio Fernández Fernández. Universidad Nacional de Educación a Distancia (U.N.E.D.).

Estudios Geográficos

Tomo LIX, n. ${ }^{\circ} 232$, julio-septiembre 


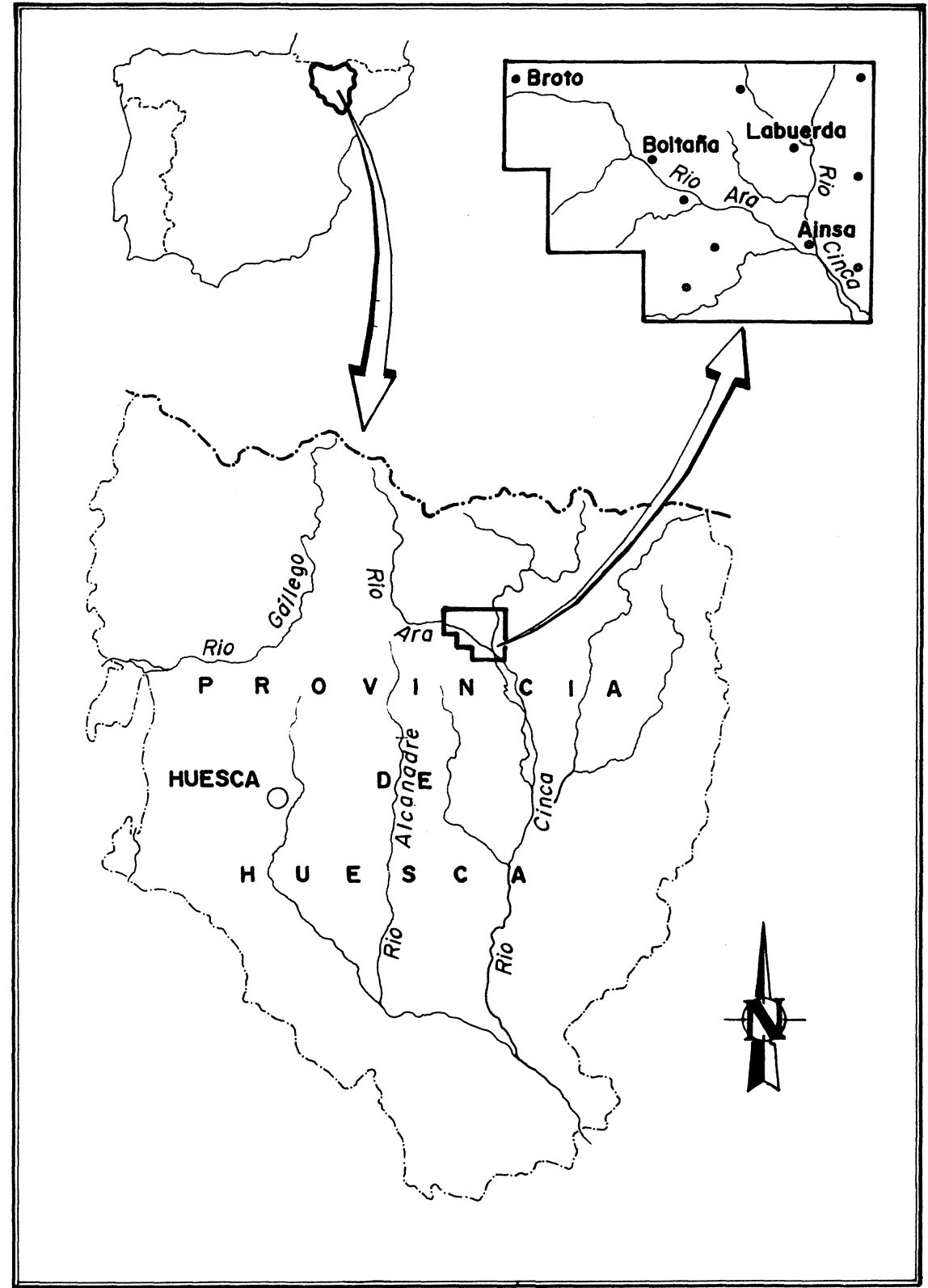

Figura 1.-Mapa de situaciónl.

$-540-$ 
que ingresan en el cauce con relativa facilidad; el río Ara, a pesar de su acentuada torrencialidad, es incapaz de evacuar toda la carga sedimentaria, lo que provoca la acumulación de materiales formando un modelado trenzado tipo «braided». Según Rust (1978a y b) este tipo de ríos son cursos de agua de múltiples canales con baja sinuosidad. Es decir, se caracterizan por la existencia de canales e islas (barras fluviales) a través de los cuales converge y diverge el flujo hídrico. Los cursos «braided» se forman en áreas de drenaje con descargas muy variables y con cubierta vegetal escasa. Geomorfológicamente, muchas a partir de aluvionamientos y sistemas «braided» (Rubio, V. y Hernández, C. 1990).

Las características más importantes que favorecen que el río Ara se comporte, en muchos tramos, como el modelo anteriormente expuesto son:

- Los fuertes desniveles obligados a salvar desde su cabecera $(3.303 \mathrm{~m})$ hasta el fondo del valle $(520 \mathrm{~m})$.

- La fácil erosionabilidad de las orillas compuestas casi siempre por material detrítico grueso (cantos y gravas) lo cual facilita el ensanchamiento y la pérdida de profundidad del canal.

- El régimen nivo-pluvial y el clima que proporcionan una circulación hídrica irregular.

La vegetación ripícola está frecuentemente condicionada por el aporte de materiales procedente de las laderas y la dinámica del río Ara. La acumulación de sedimentos y la variación de la lámina de agua permite establecer diferentes ambientes biogeográficos en las márgenes del río. Esta interacción natural (sedimentogénesis, hidrodinámica, y vegetación), se ve en algunos puntos modificada por la acción antrópica.

\section{Unidades ambientales de la vegetación ripícola}

La vegetación del fondo de valle está condicionada por los procesos geomorfológicos del lecho fluvial y la intervención humana en ciertos sectores del río. Se han establecido y estudiado cinco unidades geoambientales (figura 2) en función de la granulometría, consolidación del material sedimentado, situación respecto al cauce, grado de edafización y colonización vegetal. Estas unidades no siempre se presentan de una forma continua, lo más frecuente es que se encuentren mezcladas configurando un variado mosaico biogeográfico. 


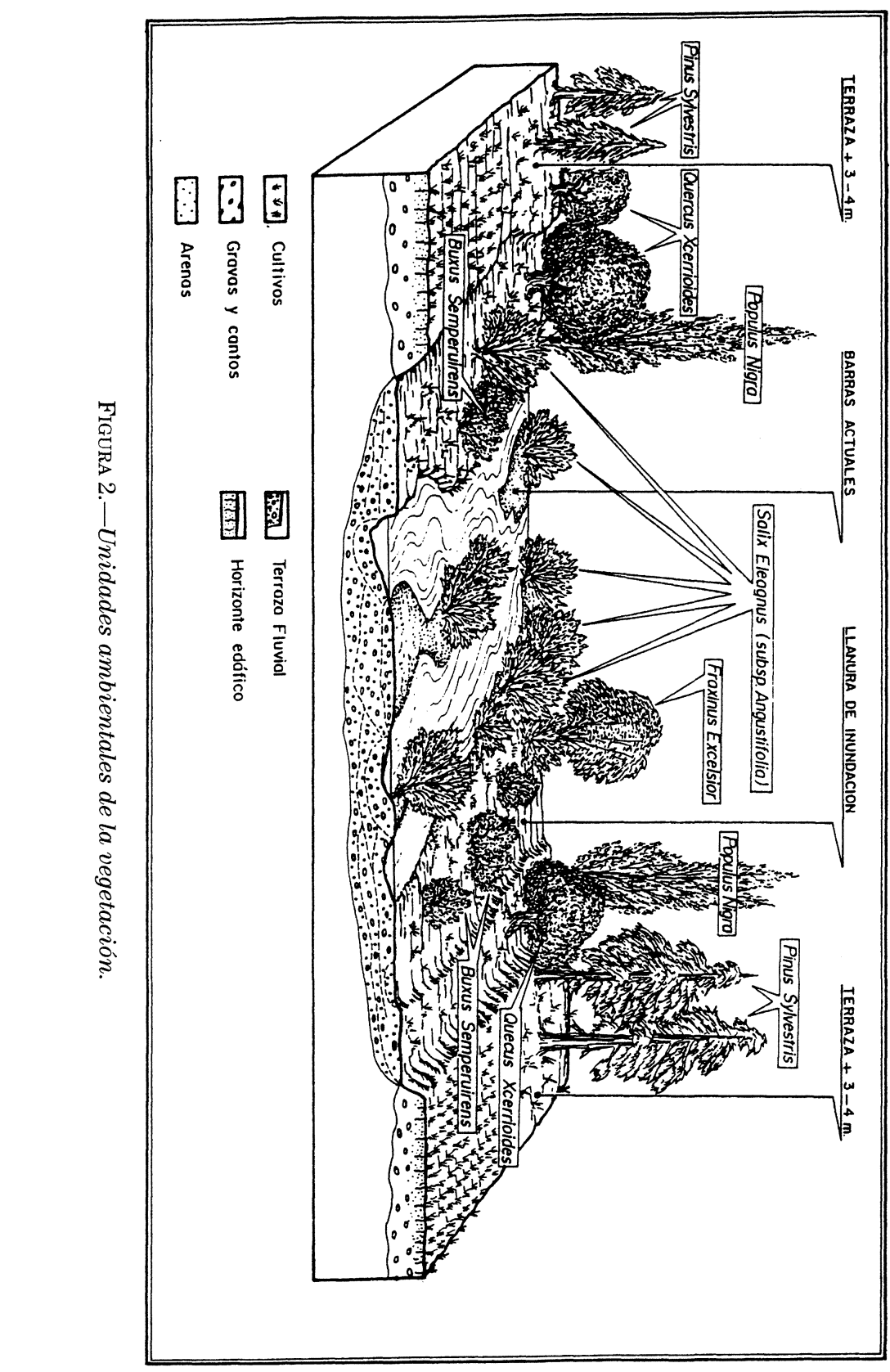

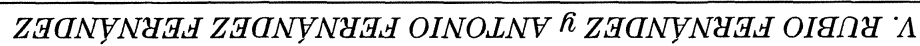


Unidades desarrolladas sobre barras fluviales.-La formacion de las barras fluviales según Leopold, Wolman y Miller (1964) se inicia con la sedimentación de los materiales más gruesos de la carga de fondo (cantos y gravas) que transporta la corriente en la parte central del canal; posteriormente, al existir un obstáculo, se van acumulando más cantos pudiendo llegar a emerger sobre el nivel del agua.

Otro tipo de procesos que puede favorecer la constitución de las barras es que el flujo hídrico no sea uniforme, esto sucede en lugares donde los canales se hacen más anchos, se arquean o en la unión de canales con distinta profundidad.

En las barras se diferencian distintos ambientes granulométricos (figura 3). En la cabeza de la barra (es decir, aguas arriba) se depositan los materiales más gruesos (gravas y cantos) debido a una pérdida de competencia de la corriente que ve disminuida su velocidad por la diferencia del flujo al chocar con la barra. Esto supone el abandono de la

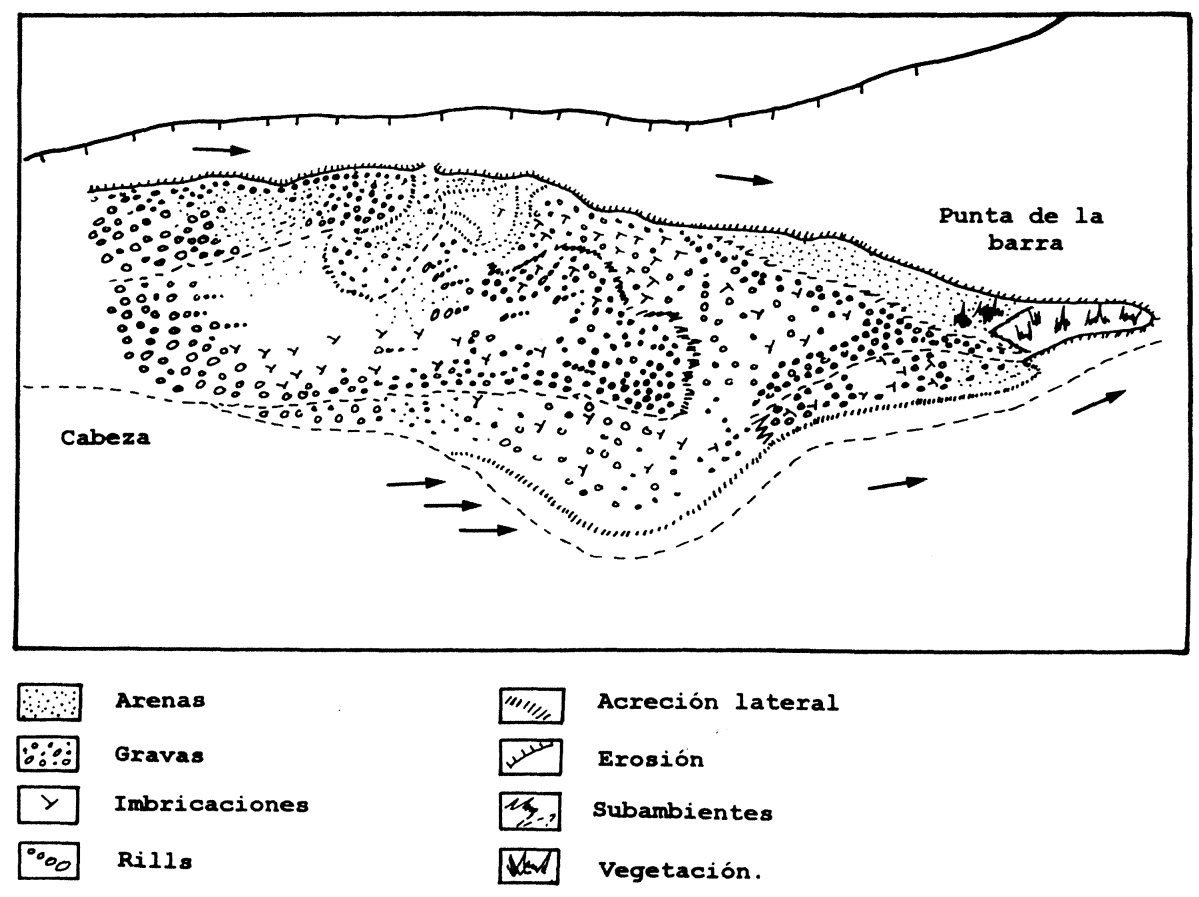

FiguRA 3.-Vista en planta de una barra actual. 
fracción gruesa (cantos y gravas) y el transporte hacia la punta de la barra (aguas abajo) de los sedimentos más finos. Como consecuencia de ello se crean ambientes granulométricamente distintos que dan lugar a la distinta colonización vegetal. Ésta comienza en la punta de la barra debido a su mayor estabilidad y a las, potencialmente, mejores condiciones edáficas que propician los limos y arcillas. En este lugar son predominante los pies de Populus nigra y Salix eleagnus subsp. angustifolia. Mientras que en la cabecera de la barra las características granulométricas (gravas y cantos) y la mayor fuerza de la corriente no permiten una colonización tan efectiva y rápida como en la punta.

Comunidades vegetales sobre graves.-Esta unidad se caracteriza por la presencia de gravas masivas con textura no granosostenida (matrix support) y por tanto sin formar ningún tipo de estructura sedimentaria (facies Gms según Miall, 1977). La escasa cobertura vegetal, en ocasiones, está sujeta a grandes crecidas y variaciones de la lámina de agua en el cauce.

Las gravas y cantos están sometidas a fuertes oscilaciones térmicas diarias especialmente en la estación estival, lo que unido, a la conservación de humedad en el subsuelo, crea un geotopo.

Las especies que colonizan las barras de gravas son refugio de gran cantidad de plantas xerófilas, muchas de ellas mediterráneas, que ascienden por los lechos fluviales, hasta latitudes muy superiores a las que suelen encontrarse. Entre otras están: Scrophularia canina, Thymus vulgaris, Plantago sempervirens, Artemisia campestris, cf. Subsp. glutinosa, Genista scorpius. Esta última siempre en barras no funcionales desde hace bastante tiempo, debiéndose su asentamiento más a su carácter heliófilo que como representativas de estas comunidades xerófilas.

Dentro de esta unidad, además, se han podido distinguir dos subambientes:

a) Pequeñas áreas donde se ha formado una capa de suelo, sujeta a gran oscilación hídrica a lo largo del año y en donde suelen encontrarse: musgos, líquenes (amortiguadores de tal oscilación) y también a veces anuales de ciclo corto, geófitas, etc.

b) Lugares que retienen algo de humedad en el suelo y en donde, con fuertes oscilaciones térmicas diarias en primavera y otoño, se producen fenómenos de movilización y turbación edáfica, apareciendo 
plantas adaptadas (Villar, F. 1977). Ambos microambientes pueden presentarse juntos y con plantas comunes.

Comunidades vegetales sobre limos y arenas finas.-La sedimentación de la fracción fina tiene lugar en las márgenes del río, puntas de las barras y en zonas donde algún tipo de obstáculo (troncos, bloques...) permiten la retención y decantación de las arenas finas, arcillas y limos. En ocasiones, la presencia de una forma arbustiva compuesta por Salix eleagnus y Populus nigra sirve para fitoestabilizar y proteger con su sistema radicular los materiales finos acumulados por el río. Estos depósitos se caracterizan por una elevada retención de humedad, así como, por su movilidad.

La vegetación que coloniza estos materiales está sometida a una fuerte inestabilidad derivada de las frecuentes avenidas del río. Por dicha razón, suelen instalarse plantas caracterizadas por una estructura radicular amplia y mínimo porte aéreo.

Entre la vegetación más abundante se encuentran gramíneas: Agrotis stolonifera, Festuca rubro y $F$. arundinacea (con menor presencia).

Unidades desarrolladas sobre canales de curso intermitente.-Las variaciones de caudal del río y del nivel freático suponen la existencia de sectores inundados ocasionalmente coincidiendo con canales abandonados en las barras, brazos inactivos del propio cauce, etc.

Aparecen comunidades vegetales compuestas por especies muy exigentes en humedad, incluso colonizan la lámina de agua, entre otras: Phragmites communis, Typha latifolia, Typha angustifolia, Juncus subnodulosus, Juncus glaucas (en inundaciones temporales).

En zonas más o menos pantanosas, la comunidad se enriquece con la presencia de Molina coelurea, acompañada de Schoenus nigricans, Scirnus pauciflorus, Scornus palustris, etc.

Unidades desarrolladas sobre la llanura de inundación (salgeral).-Esta unidad sólo se inunda en época de lluvias torrenciales o grandes avenidas. Está compuesto por un material limo-arcilloso sin estructura sedimentaria con abundantes restos vegetales (facies Fr según Miall, 1977).

La cobertura vegetal es más densa que en las anteriores unidades debido a que los sedimentos retenidos por los troncos y raíces de los sauces, consiguen independizar cada vez más a la vegetación de las 
avenidas del río, lo cual puede significar que este ambiente sea una etapa inicial o intermedia hacia la formación del bosque de ribera situado en unidades fluviales no funcionales (terraza $+3-4 \mathrm{~m}$ ).

Las especies arbóreas que predominan son: Salix eleagnus subsp. angustifolia, Populus nigra (ejemplares jóvenes) y Salix purpurea, este último en menor proporción. También se encuentra pequeños setos de Clematis vitalba, Silene vulgaris, cf. subsp. glareosa-postrata o arbustos de carácter más occidental como Genista scorpius.

Unidad de terraza baja (3-4 $\mathrm{m}$ ).-La terraza 3-4 m tiene una edad Holocea-Pleistocena superior (Rubio, V., 1995). La acumulación de los sistemas deposicionales «braided» en niveles antiguos, da lugar a una arquitectura fluvial de cuerpos mayoritariamente tabulares aunque en ocasiones se percibe cierta imbricación en las gravas. El material tiene una disposición caótica con abundancia de cantos muy gruesos; incluso en ocasiones se observan bloques de flysch arrancados del substrato. Apenas existe matriz entre los cantos (Facies Gm y Gms según Miall, 1977).

En esta unidad se asienta generalmente el bosque de ribera, que en algunos tramos del río coloniza, incluso, la llanura de inundación. Ello tiene lugar cuando la progresiva disminución en la frecuencia de avenidas supone un mayor grado de desarrollo de las comunidades vegetales de porte arbóreo. La sombra de los árboles y la capacidad higrófila del suelo permite el asentamiento de especies ombrófilas.

Choperas colonizadas por: Populus nigra, Salix eleagnus subsp. angustifolia (este último no tan dominante como en la unidad anterior). Se encuentran también otras especies propias de la chopera natural Rubus caesius, Clematis vitalba, Quercus xcerroides, Cornus sanguinea, Frangula alnus, etc

También aparecen ciertos árboles de enorme interés pero con carácter excepcional como Fagus sylvática, Acer campestre, Corylus avellana, Tilia platyphyllos entre otras; su presencia en esta unidad se debe a la caída de semillas procedente de las laderas cercanas.

La intervención humana en las áreas ribereñas, se centra en la explotación silvopastoril y más recientemente en la agricultura (árboles frutales, cereales), así como, en la construcción de embalses y diques que encauzan las aguas fluviales. Ello modifica la hidrodinámica natural 
del río, con la consiguiente degradación de las comunidades vegetales. Los incendios en el bosque de ribera y el sobrepastoreo hace que muchas veces la comunidad esté invadida por especies más pioneros: Pinus sylvestris, Buxus sempervirens y Rosa gr. Canina.

Cabe decir también que existen algunas especies comunes a todos las unidades como el Salix eleagnus subsp. angustifolia (cuadro I). Efec-

\section{Cuadro I}

INVENTARIO DE LA PRESENCIA DE ESPECIES

EN LAS DIFERENTES UNIDADES: BARRA FLUVIAL, LLANURA DE INUNDACIÓN Y TERRAZA (+3-4 M)

\begin{tabular}{|c|c|c|c|}
\hline Especies & $\begin{array}{c}\text { Barra } \\
\text { fluvial }\end{array}$ & $\begin{array}{l}\text { Llanura } \\
\text { inundación }\end{array}$ & $\begin{array}{c}\text { Terraza } \\
\text { baja }\end{array}$ \\
\hline $\begin{array}{l}\text { Salix eleagnus subsp. angustifolia } \\
\text { Populus nigra } \\
\text { Pinus sylvestris } \\
\text { Galeopsis angustifolia } \\
\text { Scrophularia canina } \\
\text { Chaenorhinum minués } \\
\text { Saponaria officinales } \\
\text { Polygonum persicaria } \\
\text { Clematis vitalba } \\
\text { Tussilago farfara } \\
\text { Verbascum thapus } \\
\text { Solanum dulcamara } \\
\text { Mentha longifolia } \\
\text { Santureja montana } \\
\text { Santureja minor } \\
\text { Asperula cynanchica } \\
\text { Gallium lucidum } \\
\text { Santolina chamaecyparissus } \\
\text { Genista scorpius } \\
\text { Ptychotis saxifraga } \\
\text { Andryala ragusina } \\
\text { Achnatherum calamagrotis } \\
\text { Thymus vulgaris } \\
\text { Buxus sempervirens } \\
\text { Rumex scutatus } \\
\text { Epatorium cannabinum } \\
\text { Holcus lanatus } \\
\text { Euphorbia nicaensis } \\
\text { Agrostis stolonifera }\end{array}$ & $\begin{array}{l}3 \\
3 \\
2 \\
1 \\
1 \\
+ \\
+ \\
1 \\
+ \\
+ \\
+ \\
1 \\
1 \\
\\
+ \\
+ \\
1 \\
+ \\
3 \\
3 \\
3\end{array}$ & $\begin{array}{l}1 \\
1 \\
1 \\
+ \\
\\
2 \\
2 \\
2 \\
2 \\
+ \\
2\end{array}$ & $\begin{array}{l}4 \\
4 \\
3\end{array}$ \\
\hline
\end{tabular}


CUADRo I (continuación)

\begin{tabular}{|c|c|c|c|}
\hline Especies & $\begin{array}{c}\text { Barra } \\
\text { fluvial }\end{array}$ & $\begin{array}{c}\text { Llanura } \\
\text { inundación }\end{array}$ & $\begin{array}{c}\text { Terraza } \\
\text { baja }\end{array}$ \\
\hline $\begin{array}{l}\text { Lythrum salicaria } \\
\text { Linum campanulatum } \\
\text { Scirpus holoschoenus } \\
\text { Policaria dissenterica } \\
\text { Equisetum palustre } \\
\text { Artemisa alba } \\
\text { Gypsophila repens } \\
\text { Plantago sempervirens } \\
\text { Plantago lanceolata } \\
\text { Oninis natrix } \\
\text { Fumaria procumbens } \\
\text { Cichorium intybus } \\
\text { Festuca gr. indigesta } \\
\text { Corylus avellana } \\
\text { Frangula alnus } \\
\text { Kileria vallesiana } \\
\text { Rubus caesius } \\
\text { Cornux sanguinea } \\
\text { Quercus xcerrioides } \\
\text { Fagus sylvestris } \\
\text { Acer campestre } \\
\text { Helleborus foetidus } \\
\text { Lonicera xylosteum } \\
\text { Viburnum lantana } \\
\text { Tillia platyphyllos } \\
\text { Sambucus nigra } \\
\text { Cronila emerus } \\
\text { Rosa canina } \\
\text { Poa nemoralis } \\
\text { Ligustrum vulgare } \\
\text { Torilis japonica } \\
\text { Brachypodium sylvaticum } \\
\text { Crataegus monogyna } \\
\text { Fraxinus excelsior }\end{array}$ & $\begin{array}{l}+ \\
+ \\
+ \\
1 \\
+ \\
+ \\
1 \\
1\end{array}$ & $\begin{array}{l}1 \\
1 \\
+ \\
1 \\
+ \\
+ \\
1 \\
+ \\
1 \\
+ \\
1\end{array}$ & $\begin{array}{l}1 \\
1 \\
2 \\
2 \\
1 \\
+ \\
1 \\
1 \\
2 \\
1 \\
+ \\
+ \\
+ \\
1 \\
1 \\
+ \\
1 \\
2 \\
2 \\
1\end{array}$ \\
\hline
\end{tabular}

$+=$ presencia

$1=$ cobertura menor de $6,5 \%$

2 = cobertura entre $6,5 \%-12,5 \%$

$3=$ cobertura entre $12,5 \%-25 \%$

$4=$ cobertura entre $25 \%-50 \%$

$5=$ cobertura superior a $50 \%$

Estos intervalos han sido elegidos en función de la representatividad de la especie. 
tivamente, son especies que han logrado un mayor grado de adaptación a los ambientes aquí referidos. En el caso de los sauces, la elasticidad de sus troncos permite soportar las fuertes avenidas de agua, que desenraizarían a otras especies leñosas más rígidas. Por otra parte, sus tallos y raíces tienen la resistencia suficiente como para convertir a la planta en un auténtico «microdique» natural (García y Fanch, 1978; Robison y Beschta, 1990; Gregory y Davis, 1992); organizando en torno a ella la sedimentación de gravas (primero) y arenas y limos (después), las cuales permitirán, a su vez, el asentamiento de nuevas especies (Pautou et al. 1985a y b).

\section{Consideraciones finales}

1. Las riberas del río Ara, en los tramos estudiados de Broto y Aínsa, constituyen biotopos sometidos a fuertes alteraciones tanto naturales (avenidas) como humanas. La inestabilidad del río, impone a las comunidades que sobre él se asientan, una dinámica rápida y compleja. En ella existe una estrecha interacción entre la vegetación y la morfología del río, que se condicionan mutuamente y que tienen como base el siguiente ciclo: erosión del río -instalación de vegetación anual o primaria - sedimentación de materiales (gravas y limos) - asentamiento de comunidades superiores - cambio en el régimen fluvial (nuevas avenidas) —nueva erosión.

2. Las plantas mejor adaptadas en todos los ambientes son Salix eleagnus subsp. angustifolia y Populus nigra teniendo gran importancia en la morfogénesis por su efecto fitoestabilizador. Sería conveniente tener en cuenta el interés de estas especies en posibles acciones de retención y colonización de ciertos tramos del curso del río.

3. En lo referente a la dinámica vegetal cabe establecer que la colonización comienza en los ambientes de barras fluviales y si las avenidas del río no causan un efecto exportador excesivo, ésta podría evolucionar hacia la unidad de salgueral y de ahí pasar al bosque de ribera.

4. Por otra parte, cabe admitir también, un proceso inverso, es decir, que desde las unidades de bosque de ribera y salgueral se retroceda a las unidades primarias, debido a la intervención humana (pastoreo, fuego). De esto se deduce que la dinámica vegetal está sometida 
a una relación bidimensional: en sentido progresivo (desde comunidades inferiores a superiores) y en sentido regresivo, básicamente condicionado por la intervención humana.

\section{BIBLIOGRAFÍA}

GARCíA, R. y FRANCH, J. (1978): «Aportación al estudio de las comunidades vegetales de ribera en el Alto Aragón Occidental». Public. de orden interior Centro Pirenaico de Biología Experimental, n. ${ }^{\circ} 22,33$ pp.

Gregory, K. J. y Davis, R. J. (1992): «Coarse Woody Debris in Stream Channels in Relation to River Channel Management in Woodland Areas». Regulated Rivers, vol. 7, pp. 117-136.

Leopold, L. B.; Wolman, M. G. y Miller, J. P. (1964): Fluvial Processes in Geomorphology. San Francisco, Freeman Comp., 522 pp.

MiALL, A. D. (1977): «A review of braided river depositional environment». Earth Science Reviews, 13, pp. 1-62.

Pautou, G.; DeCAMPS, H.; Amorós, C. y Bravard, J. P. (1985a): «Successions végétales dans les couloirs fluviaux: l'example de la plain alluviale du Haut-Rhône français». Bull. Ecolg., 16, pp. 3, 203-212.

PAUTOU, G. y DECAMPS, H. (1985b): «Ecological interactions between the alluvial forests and hydrology of the upper Rhône». Archiv. f. Hydrobiol., 104, pp. 13-37.

RoBison, E. G. y BESCHTA, R. L. (1990): «Coarse woody debris and channel mophology interactions for undisturbed streams in southeast Alaska, U.S.A.». Eartch Surface Processes and Landforms, 15, pp. 149-156.

RuBio FERNÁNDEZ, V. y HERNÁNDEZ SANTÓN, C. (1990): «La evolución reciente del cauce del río Ara». Cuadernos de Investigación Geográfica, tomo XVI, pp. 99-108.

Rubio Fernández, V. (1995): Dinámica fluvial del río Ara (Pirineo Aragonés). Tesis Doctoral, Universidad Autónoma de Madrid, 615 pp.

Rust, B. R. (1972a): «Structure and process in a braided river». Sedimentology, vol. 18, pp. 221-245.

- (1972b): «Pebble orientation in fluvial sediments». J. Sediment. Petrol., vol. 42, pp. 384-388. 